\title{
A problemática do quadrado perdido e sua relação geométrica com a sequência de Fibonacci
}

\author{
Luana Paula Goulart de Menezes(D) Vitor Marques Pereira (D)
}

\begin{abstract}
Neste artigo investigaremos o enigma do quadrado perdido (puzzle missing square) e sua relação com a sequência de Fibonacci. Acreditamos que a exploração do problema tem o potencial de relacionar diversos conteúdos dentro da própria matemática e, consequentemente, pode ser um meio profícuo para o processo de ensino e aprendizagem nos mais diferentes níveis.

Palavras-chave: O quadrado perdido; Paradoxo de Curry; Identidade de Cassini; Geometria.

Abstract

In this article we will investigate the missing square puzzle and its relation to the Fibonacci sequence. We believe that the exploration of the problem has the potential to relate different contents within mathematics itself and, consequently, can be a useful means for the teaching and learning process at the most different levels.
\end{abstract}

Keywords: The lost square; Curry Paradox; Cassini's identity; Geometry.

\section{Introdução}

O puzzle missing square conhecido como paradoxo de Curry (que não é o paradoxo de Curry da teoria dos conjuntos) é um tipo de charada geométrica que consiste em observarmos duas organizações com 4 peças geométricas planas que estão ilustradas na Figura 1 .

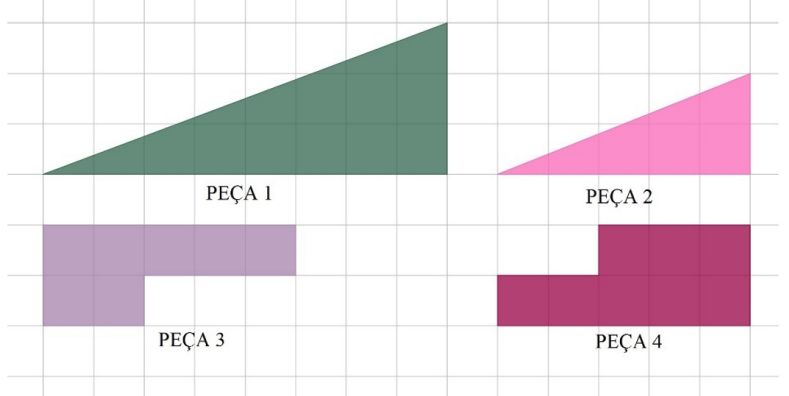

Figura 1: As 4 peças do puzzle missing square. Imagem reconstruída a partir de [2]. 
Usando as peças ilustradas acima, vamos organizá-las de modo a obter a Figura 2 que reproduzimos abaixo:

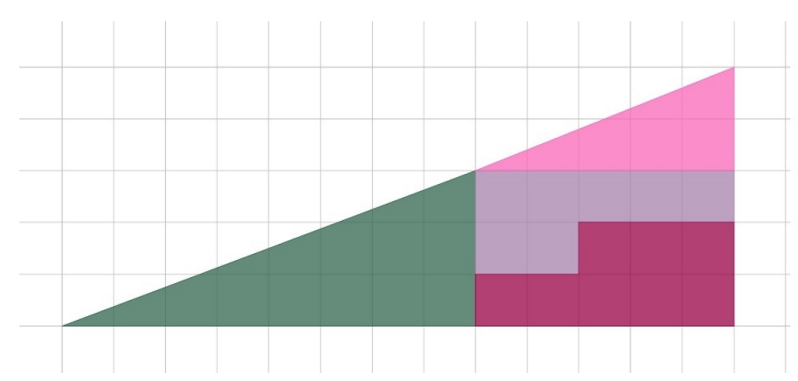

Figura 2: Organização das peças. Imagem reconstruída a partir de [2].

Observe que a figura obtida parece se ajustar ao triângulo retângulo com dimensões $13 \times 5$. Note ainda que podemos reorganizar as posições das peças como mostra a Figura 3:

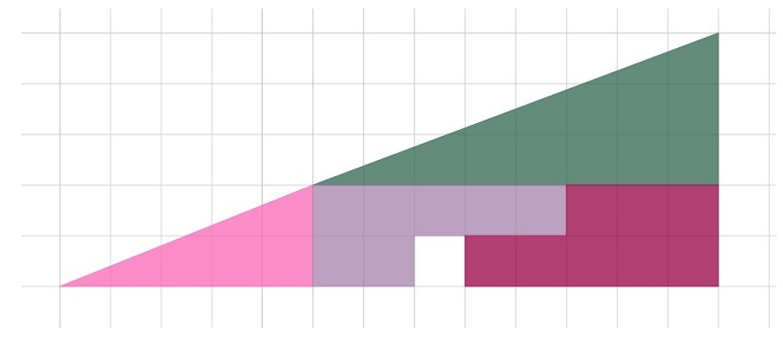

Figura 3: O quadrado perdido. Imagem reconstruída a partir de [2].

Perceba que tal disposição das peças também parece se ajustar em um triângulo retângulo com dimensões $13 \times 5$, com uma diferença: falta um quadrado! Como usamos as mesmas peças, deveríamos ter a conservação da área em ambas as disposições. Então, como isso é possível? Onde está o quadrado? O objetivo de apresentarmos o puzzle é responder essas perguntas.

\section{A Área das peças e a área do "triângulo" construído}

Com o objetivo de explicar o que aconteceu com o quadrado, vamos começar verificando a área de cada peça, encontrando, assim:

Peça 1: $\frac{8 \cdot 3}{2}=12$ u.a.

Peça 2: $\frac{5 \cdot 2}{2}=5$ u.a.

Peça 3: $1 \cdot 5+1 \cdot 2=7$ u.a.

Peça 3: $1 \cdot 3+1 \cdot 5=8$ u.a.

Desse modo, temos que área total ocupada pelas peças é: 


$$
\mathrm{A}=12+5+7+8=32 \text { u.a. }
$$

Mas como em ambas as Figuras 2 e 3 formamos um triângulo retângulo de dimensões $13 \times 5$, temos que área do mesmo pode ser obtida fazendo:

$$
\mathrm{A}=\frac{13 \cdot 5}{2}=32,5 \text { u.a. }
$$

Logo, as peças 1, 2, 3 e 4 não cobrem todo o triângulo retângulo, pois não preenchem meio quadrado. Contudo, note que estamos observando a falta de meio quadrado e não 1 quadrado. Para explicarmos qual o segredo da disposição das peças, vamos calcular o ângulo $\theta$ entre a hipotenusa e o cateto adjacente das peças 1 e 2 e do triângulo obtido com a arrumação das peças que chamaremos de $\mathrm{T}$ ( com catetos 13 e 5):

Peça 1: $\theta=\arctan \left(\frac{3}{8}\right) \approx 20,56^{\circ}$

Peça 1: $\theta=\arctan \left(\frac{2}{5}\right) \approx 21,8^{\circ}$

$\mathbf{T}: \theta=\arctan \left(\frac{5}{13}\right) \approx 21,04^{\circ}$

Disso, obtemos que a hipotenusa não é uma reta, pois da construção realizada deveríamos obter o mesmo valor para o ângulo $\theta$. Portanto, as figuras obtidas não são triângulos retângulos e sim quadriláteros. Mas como não notamos isso nas duas imagens? Uma explicação plausível está na diferença pequena entre os declives das peças 1 e 2 :

$$
\frac{2}{5}-\frac{3}{8}=\frac{1}{40}
$$

Logo, a diferença ínfima não nos permite visualizá-la. As figuras 4 e 5 fora de escala ilustram o detalhe de falta e sobra nas áreas:

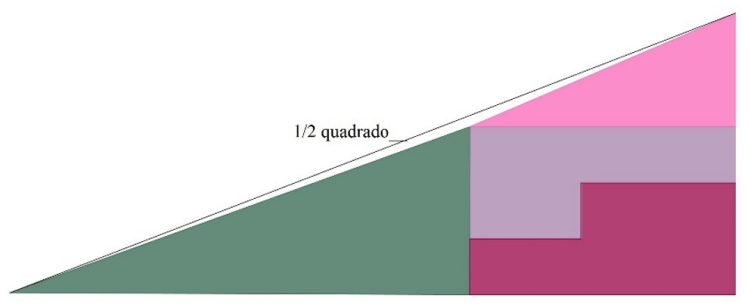

Figura 4: O quadrado perdido. Imagem reconstruída a partir de [2]. 


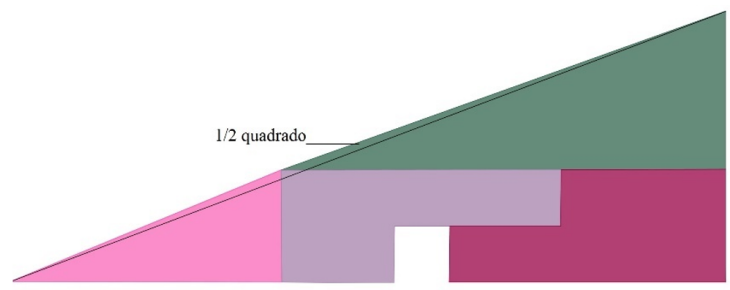

Figura 5: Visualizando a diferença. Imagem reconstruída a partir de [2].

Assim, o quadrado perdido está na metade de um quadrado que falta na Figura 4 e na metade do quadrado que sobra na Figura 5

\subsection{A identidade de Cassini}

Vamos observar ainda algo interessante: as dimensões inteiras dos lados das peças são 1, 2, 3, 5, 8 e 13, termos consecutivos da sequência de Fibonacci, isto é, $8 \mathrm{n} \geq 3, \mathrm{f}_{\mathrm{n}}=\mathrm{f}_{\mathrm{n}-1}+\mathrm{f}_{\mathrm{n}-2}$, com $\mathrm{f}_{1}=1$ e $\mathrm{f}_{2}=1(\operatorname{ver}[3])$.

Para efeito de curiosidade, aqui cabe um pequeno parêntese sobre Fibonacci (c.1175-c.1240): sabemos que nasceu em Pisa, Itália, e que "[...] viveu parte de sua juventude no norte da África, onde aprendeu o idioma e familiarizou-se com os costumes e a cultura árabes" (Santos, 2018, p. 20). Além disso, "Viajou extensamente pelo Mediterrâneo durante grande parte de sua vida, o que lhe rendeu o apelido Leonardo Bigollo (Leonardo Viajante). De volta à Itália, em torno de 1200, publicou o Liber Abaci (Livro do Ábaco ou Livro de Cálculo) em 1202" (Santos, 2018, p. 20). Em tal livro ele apresentou uma situação hipotética acerca da reprodução de coelhos, que é amplamente conhecido (veja mais em [3]).

No mais, vejamos que é uma propriedade da sequência citada acima que o produto de dois termos alternados da sucessão difere de uma unidade do quadrado do termo intermediário, isto é,

$$
f_{n-1} \cdot f_{n+1}=f_{n}^{2}+(-1)^{n}, 8 n \in \mathbb{N} .
$$

Exemplo 1: Para visualizarmos a relação escolhemos como exemplo os números consecutivos da sequência: 2 , 3 e 5 . Dessa forma: $2 \cdot 5=10$ e $3^{2}=9$, que satisfaz (1) com a adição do número 1 no segundo membro.

Exemplo 2: Do mesmo modo, se escolhermos os números 3,5 e 8 , obteremos $3 \cdot 8=24$ e $5^{2}=25$, que também satisfaz (1), agora com a subtração do número 1 no segundo membro.

Tal propriedade é conhecida como identidade de Cassini. Para demonstrá-la vamos considerar a matriz $F=\left[\begin{array}{ll}1 & 1 \\ 1 & 0\end{array}\right]$ e $f_{n+2}=f_{n+1}+f_{n}, c_{0}=0$ e $f_{1}=1$ a sequência de Fibonacci.

Note que estamos considerando o primeiro termo como sendo zero, mas isso não altera a sequência e suas propriedades.

Assim, temos que se $\mathrm{F}=\left[\begin{array}{ll}1 & 1 \\ 1 & 0\end{array}\right]$ : 


$$
\begin{gathered}
\mathrm{F}^{2}=\left[\begin{array}{ll}
1 & 1 \\
1 & 0
\end{array}\right] \cdot\left[\begin{array}{ll}
1 & 1 \\
1 & 0
\end{array}\right]=\left[\begin{array}{ll}
2 & 1 \\
1 & 1
\end{array}\right] \\
\mathrm{F}^{3}=\left[\begin{array}{ll}
2 & 1 \\
1 & 1
\end{array}\right] \cdot\left[\begin{array}{ll}
1 & 1 \\
1 & 0
\end{array}\right]=\left[\begin{array}{ll}
3 & 2 \\
2 & 1
\end{array}\right] \\
\mathrm{F}^{4}=\left[\begin{array}{ll}
3 & 2 \\
2 & 1
\end{array}\right] \cdot\left[\begin{array}{ll}
1 & 1 \\
1 & 0
\end{array}\right]=\left[\begin{array}{ll}
5 & 3 \\
3 & 2
\end{array}\right] \\
\mathrm{F}^{\mathrm{n}}=\left[\begin{array}{cc}
\mathrm{f}_{\mathrm{n}+1} & \mathrm{f}_{\mathrm{n}} \\
\mathrm{f}_{\mathrm{n}} & \mathrm{f}_{\mathrm{n}-1}
\end{array}\right] .
\end{gathered}
$$

Para provarmos a última igualdade, vamos usar o Princípio de Indução Finita; mostrando que a igualdade é válida para $\mathrm{n}=1$, e, supondo que é válida para $\mathrm{n}=\mathrm{k}$, iremos mostrar que vale para $\mathrm{n}=\mathrm{k}+1$ :

i) F é válida para $\mathrm{n}=1$, pois:

$$
F^{1}=\left[\begin{array}{ll}
f_{2} & f_{1} \\
f_{1} & f_{0}
\end{array}\right]=\left[\begin{array}{ll}
1 & 1 \\
1 & 0
\end{array}\right]
$$

ii) Suponha que $\mathrm{F}$ é válida para $\mathrm{n}=\mathrm{k}$, então:

$$
F^{k}=\left[\begin{array}{cc}
f_{k+1} & f_{k} \\
f_{k} & f_{k-1}
\end{array}\right]
$$

Multiplicando ambos os membros da Equação (2) por F, temos:

$$
F^{k} \cdot F=\left[\begin{array}{cc}
f_{k+1} & f_{k} \\
f_{k} & f_{k-1}
\end{array}\right] \cdot\left[\begin{array}{cc}
1 & 1 \\
1 & 0
\end{array}\right] \Leftrightarrow F^{k+1}=\left[\begin{array}{cc}
f_{k+1}+f_{k} & f_{k+1} \\
f_{k}+f_{k-1} & f_{k}
\end{array}\right]=\left[\begin{array}{cc}
f_{k+2} & f_{k+1} \\
f_{k+1} & f_{k}
\end{array}\right] .
$$

Logo, $\mathrm{F}$ vale para $\mathrm{n}=\mathrm{k}+1$. Portanto:

$$
F^{n}=\left[\begin{array}{cc}
f_{n+1} & f_{n} \\
f_{n} & f_{n-1}
\end{array}\right]
$$

Voltando na identidade de Cassini, temos $\mathrm{f}_{\mathrm{n}-1} \cdot \mathrm{f}_{\mathrm{n}+1}=\mathrm{f}_{\mathrm{n}}^{2}+(-1)^{\mathrm{n}} \cdot \operatorname{Como} \operatorname{det}\left(\mathrm{F}^{\mathrm{n}}\right)=\mathrm{f}_{\mathrm{n}+1} \cdot \mathrm{f}_{\mathrm{n}-1}-\mathrm{f}_{\mathrm{n}}^{2}$, devemos mostrar que $\operatorname{det}\left(\mathrm{F}^{\mathrm{n}}\right)=(-1)^{\mathrm{n}}$.

E, de fato, é propriedade dos determinantes que:

$$
\operatorname{det}\left(\mathrm{F}^{\mathrm{n}}\right)=[\operatorname{det}(\mathrm{F})]^{\mathrm{n}} \text {. }
$$


Como $\operatorname{det}(\mathrm{F})=-1$, podemos substituir na Equação $(3)$ e obtermos que $\operatorname{det}\left(\mathrm{F}^{\mathrm{n}}\right)=(-1)^{\mathrm{n}}$.

Logo, $\operatorname{det}\left(F^{n}\right)=f_{n+1} \cdot f_{n-1}-f_{n}^{2}$, ou seja, $(-1)^{n}=f_{n+1} \cdot f_{n-1}-f_{n}^{2}$. Portanto:

$$
f_{n-1} \cdot f_{n+1}=f_{n}^{2}+(-1)^{n}
$$

como queríamos demonstrar.

\section{Identidade de Cassini e a relação geométrica com a construção}

Como vimos, existe uma relação entre os três termos da sequência de Fibonacci. Todavia, como isso se relaciona com nossa problemática?

Para responder essa questão, iremos usar as notações de [1]:

$$
\left\{\begin{array}{l}
A+B=C \\
B^{2}=A \cdot C \pm X
\end{array}\right.
$$

em que A, B e C, no sistema, são consecutivos da sequência de Fibonacci, e, como vimos, temos que $\pm \mathrm{X}$ na identidade de Cassini é \pm 1 (a segunda equação do sistema). Notamos então, que temos a área de um quadrado de lado B que ao compararmos com o lado de um retângulo com lados $\mathrm{A}$ e $\mathrm{C}$, deveremos acrescentar ou retirar uma unidade.

No exemplo que estamos considerando desde o início deste artigo podemos dobrar as peças que estão na Figura 1 para termos um quadrado com $A=64 u$.a.. Assim, uma possível organização é ilustrada na Figura 6.

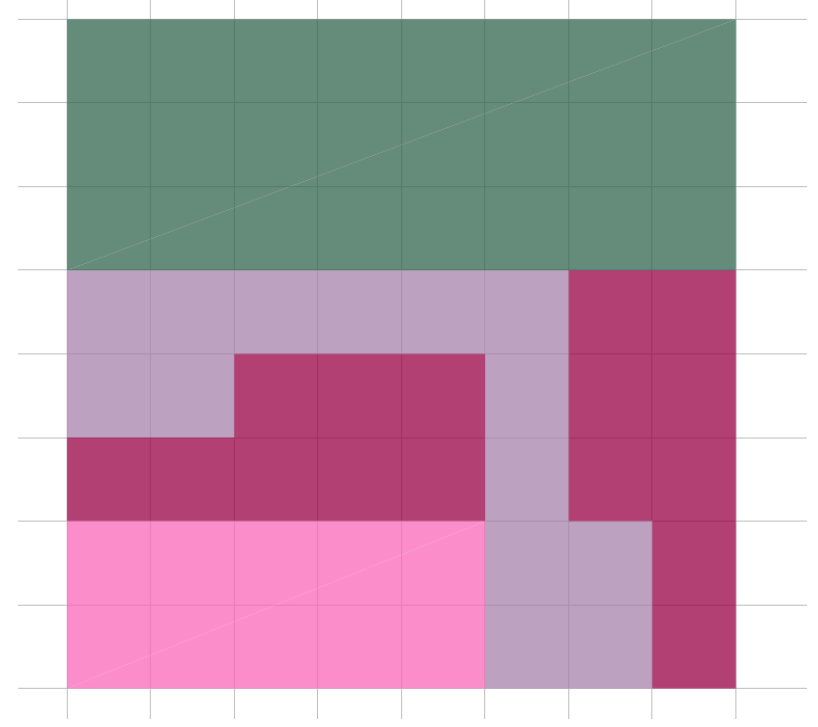

Figura 6: Uma organização quadrada das peças. 
Note agora que usando as mesmas peças teremos a falta de um quadrado (Figura 7):

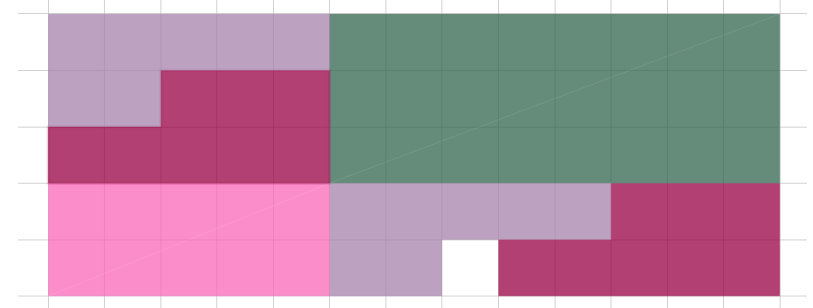

Figura 7: Uma organização retangular das peças.

Isso já era esperado, uma vez que a equação $8^{2}=5 \cdot 13+\mathrm{X}$ é válida com $\mathrm{X}=-1$. Isso significa que um quadrado é perdido de uma organização para outra.

\section{Considerações Finais}

Da identidade de Cassini temos que outras figuras que apresentam o mesmo fenômeno podem ser obtidas com termos consecutivos da sequência de Fibonacci, como o puzzle de Carroll que não foi apresentado neste artigo.

Ademais, com a motivação do enigma do quadrado perdido, podem ser ensinados vários conceitos, por exemplo, área, declive, o princípio de indução finita, determinantes, sequência de Fibonacci e propriedades. Não podemos deixar de mencionar ainda que sua construção tem a vantagem de visualização e manipulação de material concreto, sendo assim um interessante instrumento para o processo de ensino-aprendizagem. Tal problemática pode ser explorada tanto em níveis básicos como em nível superior considerando-se diversos níveis de complexidade.

\section{Referências}

[1] Gardner, M. Mathematics, magic and mystery. Dover, New York, 1956.

[2] Santos, C. P.; NETO, J. P.; SILVA, J. N. Sucessão de Fibonacci + 'Missing Square'. Lisboa: Público-Visão, 2007.

[3] Santos, F. H. Funções de Fibonacci:Um estudo sobre a razão áurea e a sequência de Fibonacci. 2018. 51 f. Dissertação (Mestrado) -Profmat, Universidade Federal de Alagoas, Maceió, 2018.

Luana Paula Goulart de Menezes Universidade Estadual de Maringá $<$ lluanagoulart@gmail.com>

Vitor Marques Pereira Universidade Estadual do Oeste do Paraná $<$ vitormarques@yahoo.com> 